\title{
Genome-wide SNP-based linkage analysis for ADNSHL families identifies novel susceptibility loci with positive evidence for linkage
}

\author{
Mi-Hyun Park ${ }^{1 \dagger}$, Hong-Joon Park ${ }^{2 \dagger}$, Kwang-Joong Kim ${ }^{3 \dagger}$, Hae-Mi Woo ${ }^{1}$, \\ Hyung-Jin Kim ${ }^{1}$, Jong-Young Lee ${ }^{3}$, Hyun-Young Park ${ }^{1}$ \\ and Soo Kyung $\mathrm{Koo}^{1 *}$ \\ ${ }^{1}$ Division of Cardiovascular and Rare Diseases, Center for Biomedical Sciences, \\ National Institute of Health, South Korea \\ ${ }^{2}$ Soree Ear Clinic, South Korea \\ ${ }^{3}$ Division of Structural and Functional Genomics, Center for Genome Science, \\ National Institute of Health, South Korea
}

(Received 25 December 2010, accepted 19 March 2011)

\begin{abstract}
The linkage search for susceptibility loci using SNP markers in hereditary hearing loss has proven challenging due to genetic heterogeneity. We conducted a genome-wide linkage analysis using high-density SNP markers in two Korean families (families coded SD-J and SR-167) with autosomal dominant nonsyndromic hearing loss (ADNSHL). Evidence was found of linkage at 8q24.13 q24.3 and 10p11.21 q22.2 (LOD 3.01) in the SD-J family. In the case of family SR-167, which had the most affected members, the parametric LOD score was low owing to the lack of power for linkage analysis. However, using nonparametric linkage analysis, it was possible to obtain significant evidence for linkage at 10q22.1 q23.31 (LOD 1.79; NPL 6.47, $P<0.00001$ ). There is an overlapping region with a significant LOD score between the SD-J and SR-167 families, which encompasses $4 \mathrm{cM}$ at 10q22.1 22.2. Interestingly, the characteristics of hearing loss in both families were similar, and the haplotype within overlapping region was shared in the affected individuals of the two families. We performed direct sequencing of the candidate genes that are thought to be causing the condition, but no disease-causing mutations were identified.
\end{abstract}

Key words: ADNSHL, SNP-based linkage, 10q22.1 q22.2, Korean

\section{INTRODUCTION}

Hearing loss is the most prevalent sensory disorder in the general population. Approximately 1 in 1,000 newborns is affected by severe to profound deafness at birth or during early childhood (Bitner-Glindzicz, 2002). Half of these cases can be attributed to heredity/genetics. For non-syndromic genetic hearing loss, $20 \%$ of cases are dominant hereditary types, whereas $80 \%$ of cases are recessive types (Cryns and Van Camp, 2004). Hearing loss is genetically highly heterogeneous, with over 110 mapped loci and 55 causally implicated genes (http:// hereditaryhearingloss.org).

Family-based genome-wide linkage analysis is widely

\footnotetext{
Edited by Hidenori Tachida

* Corresponding author. E-mail: skkoo@nih.go.kr

$\dagger$ These authors contributed equally to this work.

Note: Supplementary materials in this article are at http:// www.jstage.jst.go.jp/browse/ggs
}

used for determining the genetic basis of human hereditary disease. Traditionally, genome-wide linkage analysis involves low-density mapping of microsatellite markers spaced at intervals of $\sim 10 \mathrm{cM}$ across the genome. The recent development of single nucleotide polymorphism (SNP) technologies has facilitated a genome-wide approach to the search for novel candidate genes involved in hereditary diseases (Gonzalez-Neira et al., 2007). High-throughput genotyping of SNPs offers cost effectiveness with high accuracy, and densely distributed markers have increased the power of detection of linkages. This allows more sensitive and precise identification of disease loci (Evans and Cardon, 2004; John et al., 2004; Schaid et al., 2004). In the present report, we describe the SNPbased genome-wide linkage results for two Korean families (SD-J and SR-167) suffering from autosomal dominant non-syndromic hearing loss (ADNSHL). 


\section{MATERIALS AND METHODS}

Family ascertainment Two three-generation Korean families with ADNSHL through the probands, III:5 (SDJ) and III:2 (SR-167) (Figs. 1 and 2), were studied. The probands in both families were diagnosed with hearing impairment in their early teenage years. All the affected individuals in both families had bilateral, moderately severe sensorineural hearing loss without additional symptoms. Individuals III-5 and III-6 of the SD-J family, who are genetically monozygotic twins, were included in the linkage analysis. Genomic DNA was extracted from peripheral blood samples using the FlexiGene DNA extraction kit (QIAGEN, Hilden, Germany). Informed consent was obtained from all the participants, and this study was approved by the Institutional Review Board (IRB) at Korea NIH.

SNP genotyping and error checking SNP markers were genotyped in the individuals using the Affymetrix Genome-Wide Human SNP Array 5.0 (Affymetrix, USA).

A

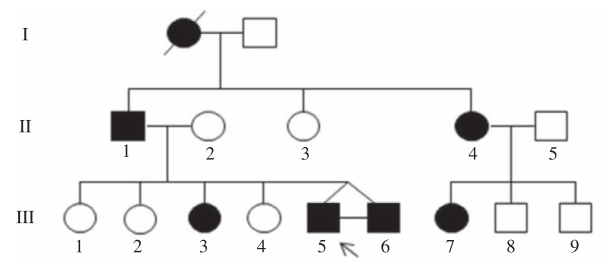

B
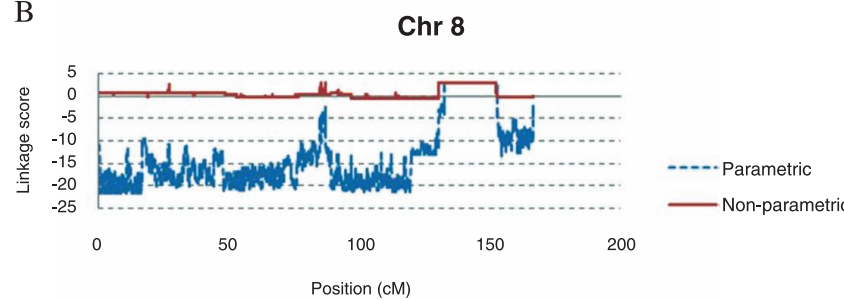

Chr 10

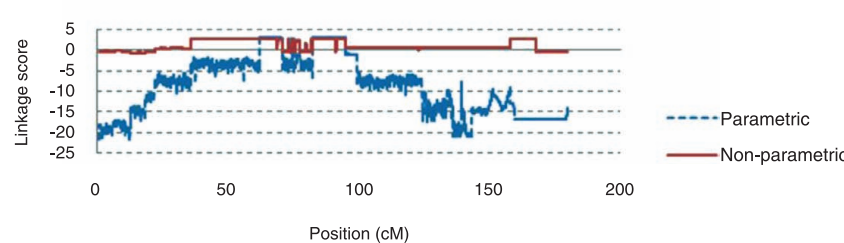

Fig. 1. Pedigree of the SD-J family and multi-point linkage analyses. (A) The pedigree of the SD-J family, including the dominant inheritance pattern of three generations, is shown. Affected individuals are represented as black symbols. Individuals with genotype data are identified by numbers under the symbols in the pedigree. (B) Multi-point linkage analyses (Chromosomes 8 and 10) of the SD-J family. Blue (dotted line) and red (full line) represent parametric linkages (LOD scores) and non-parametric linkages (NPL scores), respectively.
The assay was performed using $250 \mathrm{ng}$ of genomic DNA. Scanned images were processed with the Affymetrix Microarray Suite (MAS) software, and the data were analyzed with the GDAS v2 software. SNPs were excluded from analyses based on: 1) SNP call rates < 90\%; 2) a minor allele frequency $(\mathrm{MAF})<0.1 ; 3)$ a monomorphic allele; and 4) insufficient map information.

The PEDSTATS software was used to determine the genotyping success rate, confirm the pedigree structure, and specify the relationships between the individuals in each family (Wigginton and Abecasis, 2005). The error detection approach in the Merlin program was used to search for Mendelian inconsistencies within each of the SNP clusters, and improbable genotypes were removed using the program's wipe function. We also detected marker typing incompatibilities in the pedigree data, and all ambiguous marker genotypes were deleted.

Linkage analysis Genome-wide linkage analysis was carried out with 259,361 SNP markers (Supplementary Table S1) using the linkage program Merlin-1.1.2 (Abecasis et al., 2002). Single-point and multi-point analyses for each family were performed using ALL frequencies, and both parametric and non-parametric analyses were performed. The parametric LOD scores were computed using an assumed dominant model and a susceptibility allele with population frequency of 0.001 . Non-parametric LOD (NPL) scores were calculated using

A

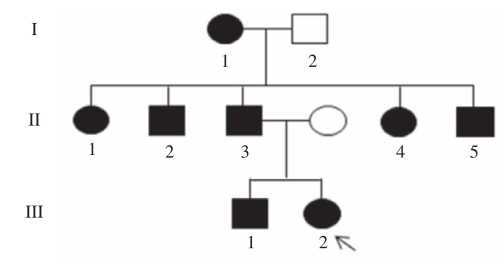

B

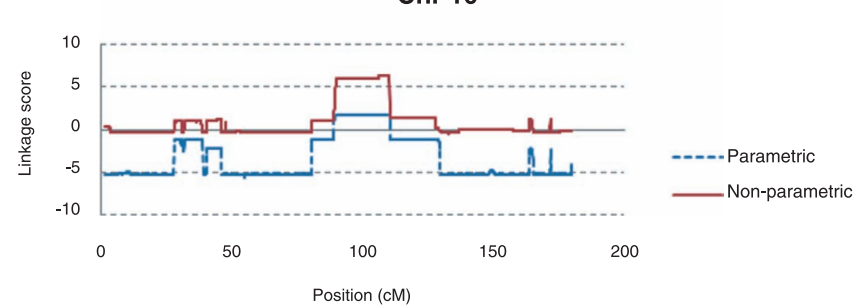

Fig. 2. Pedigree of the SR-167 family and multi-point linkage analysis. (A) The pedigree of the SR-167 family, including the dominant inheritance pattern for three generations, is shown. Affected individuals are represented as black symbols. Individuals with genotype data are identified by numbers under the symbols in the pedigree. (B) Multi-point linkage analysis (chromosome 10) of the SR-167 family. Blue (dotted line) and red (full line) represent parametric linkage (LOD score) and non-parametric linkage (NPL score), respectively. 
the exponential allele sharing of all possible informative affected relative pairs within the family. SNP marker positions and map distances were derived from data provided by deCODE Genetics (Kong et al., 2002).

To assess the impact of linkage disequilibrium (LD), we used the $r s q$ option of the Merlin software to accommodate marker-marker LD in both the parametric and nonparametric multi-point analyses. Merlin analyzes SNPs that are in LD by treating them as multi-allelic markers and employing the EM algorithm (Abecasis and Wigginton, 2005). Markers with $r^{2}$ values $>0.4$ were organized into clusters.

To assess the false-positive rate of our linkage results, we used the simulation option in the Merlin software to estimate empirically the probability that the observed proportion of the genome that showed excess allele sharing was observed by chance. This analysis replaces our real data with simulated chromosomes, while maintaining the original pedigree structure, allele frequencies, and recombination fraction and retaining the original missing data patterns. We performed the simulation analysis according to the method of North et al. (2003).

Mutation screening using sequence analyses Candidate genes on chromosome 10q22.1 22.2 were identified using the UCSC Genome Bioinformatics web browser (http://www.genome.ucsc.edu/) and were initially selected for mutation screening on the basis of expression of function in the inner ear. The primers used for PCR amplification and subsequent sequencing of PSAP and MYOZ1 were designed from the flanking region of each exon using the Primer3 web utility (http://www.frodo.wi.mit.edu/cgibin/primer3/primer3_www.cgi/). The PCR products were directly sequenced using an ABI 3730 automated sequencer and the Big Dye Terminator Sequencing Kit (Applied Biosystems, USA). Five persons with hearing loss, i.e., III-3 and III-5 of family SD-J (Fig. 1) and II-1, II-2, and II-3 of family SR-167 (Fig. 2), were investigated for the presence of mutations. Sequence comparisons were performed using the Sequencher software (Gene Codes Corp., USA).

\section{RESULTS AND DISCUSSION}

In total, 14 individuals from the SD-J family were genotyped (Fig. 1). Initial single-point analysis revealed four chromosomal regions (NPL 2.14 2.72, $P<0.01$; LOD 2.05 3) that appeared to harbor the putative ADNSHL susceptibility loci in the SD-J family (Supplementary Table S2). The multi-point linkage was also determined to accommodate the marker LD SNPs with $r^{2}$ values > 0.4. Of these four linked chromosomal regions, two regions (chromosomes 8 and 10) were identified by multipoint analyses (Fig. 1 and Supplementary Fig. S1). In chromosome region $8 \mathrm{q} 24.13 \sim \mathrm{q} 24.3$, the maximum parametric multi-point analysis yielded suggestive evidence (LOD score 3.01) for SNP markers from rs11988106 to rs17634990 (genetic map position: 132 151 cM; physical map position: 127,487,483 138,612,616) (Fig. 1 and Table 1 ). In the case of chromosome 10 , the maximum parametric multi-point analysis also yielded suggestive evidence (LOD score of 3.01) at four loci: $62 \sim 68 \mathrm{cM} ; 69 \sim 70$ cM; 82 90 cM; and 92 94 cM. These genomic regions were supported by the non-parametric linkage analysis. The maximum multi-point NPL scores for chromosome 8 and 10 were both $2.8(P=0.003)$ (Fig. 1 and Table 1$)$. To confirm our linkage result with SNP markers, we performed linkage analysis using microsatellite markers which span 63 93 cM on chromosome 10 for the SD-J family and we found the linkage evidence with LOD score of 2.7 .

In total, nine individuals from the SR-167 family were genotyped (Fig. 2). Initial single-point analysis revealed ten chromosomal regions (NPL 4.75 6.27, $P<0.00001$; LOD 1.65 1.79), representing highly significant evidence of linkage (Supplementary Table S2). Of these linked chromosomes, chromosomes 7,10 , and 20 were confirmed by multi-point analysis (Supplementary Fig. S2). The

Table 1. Multi-point linkage analyses results for each family using parametric and non-parametric methods

\begin{tabular}{|c|c|c|c|c|c|c|}
\hline \multirow{2}{*}{ Family } & \multicolumn{3}{|c|}{ Position } & \multirow{2}{*}{$\begin{array}{c}\text { Parametric analysis } \\
\text { LOD }\end{array}$} & \multicolumn{2}{|c|}{ Non-parametric analysis } \\
\hline & Chromosome & & $\mathrm{cM}$ & & NPL & $P$-value \\
\hline \multirow[t]{5}{*}{ SD-J } & 8 & $8 q 24.13 \sim q 24.3$ & $132 \sim 151$ & 3.01 & 2.80 & 0.003 \\
\hline & 10 & 10p11.21 q11.22 & $62.6 \sim 68.6$ & \multirow{4}{*}{3.01} & \multirow{4}{*}{2.80} & \multirow{4}{*}{0.003} \\
\hline & & $10 q 11.22 \sim q 11.23$ & $69 \sim 70.4$ & & & \\
\hline & & 10q21.3 22.1 & 82.4 91.2 & & & \\
\hline & & $10 \mathrm{q} 22.1 \sim \mathrm{q} 22.2$ & $91.9 \sim 94.7$ & & & \\
\hline \multirow[t]{4}{*}{ SR-167 } & 7 & $7 \mathrm{p} 22.3 \sim \mathrm{p} 21.3$ & $\sim 15$ & 1.79 & 6.09 & $<0.00001$ \\
\hline & 10 & $10 \mathrm{q} 22.1 \sim \mathrm{q} 23.31$ & $90 \sim 109$ & 1.79 & 6.47 & $<0.00001$ \\
\hline & 20 & $20 \mathrm{p} 12.1 \sim \mathrm{p} 11.21$ & $45 \sim 53$ & \multirow{2}{*}{1.79} & \multirow{2}{*}{6.11} & \multirow{2}{*}{$<0.00001$} \\
\hline & & 20q13.12 q13.33 & $68 \sim 114$ & & & \\
\hline
\end{tabular}


maximum multi-point NPL yielded significant evidence (NPL 6.47, $P<0.00001$ ) of SNP markers (genetic map position: 90 109 cM) at chromosomal region 10q22.1 q23.31 (Fig. 2 and Table 1). The two loci identified at chromosomal regions 20p12.1 p11.21 and 20q13.12 q13.33 yielded the second highest NPL value (NPL 6.11, $P<0.00001$ ). The $7 \mathrm{p} 22.3 \sim \mathrm{p} 21.3$ locus also yielded significant evidence (NPL 6.09, $P<0.00001$ ). We conducted 1,000 simulations to assess the empirical genome-wide significance level of these linkage peaks. The maximal NPL score for chromosome 10 showed marginal significance $(P=0.049)$. However, the peaks for chromosomes $7(P=0.1)$ and $20(P=0.1)$ did not reach significance (data not shown). These results indicate that the simulated data for chromosome 10 are more suggestive of allele sharing than would be expected by chance.

We identified novel genomic regions with suggestive or significant levels of evidence for ADNSHL in two Korean families. The genomic regions identified in the present study do not overlap with the reported loci for ADNSHL, although the DFNB12 (causative gene: $C D H 23$ ) and DFNB57 (unknown gene) loci have been reported in cases of autosomal recessive hearing loss. Several chromosomal regions identified by single-point analyses have been discounted as causative regions using multi-point analyses. The most interesting outcome of the present study is the evidence of linkage in the SR-167 family. As most of the members of the SR-167 family are affected, it is difficult to obtain significant results using conventional linkage analysis. Using parametric linkage analysis, a low linkage signal is obtained, as this value depends on the assumed genetic model and the power of the samples within the family. However, we identified a significant linkage signal in the same region using non-parametric linkage analysis, which was calculated using exponential allele sharing of all possible informative affected pairs within the SR-167 family. This suggests that the result of non-parametric linkage analysis generates a stronger linkage signal for a nuclear family with a low power for calculating the LOD score, as seen with the SR-167 family. These results highlight the importance of performing both parametric and non-parametric linkage analyses and of confirmation by multi-point analysis to avoid false-negative or false-positive results.

In both families, the same linkage peak on chromosome 10 spanned approximately $4 \mathrm{Mb}$ between SNPs rs 3740458 and rs839698 (Supplementary Fig. S3). This region corresponds to the cytogenetic chromosome bands 10q22.1 22.2 (UCSC Genome Browser). In the 10q22.1 22.2 linkage interval are 38 known genes, including CDH23 (DFNB12). In this region, we found that affected individuals from both families shared the haplotype blocks. This shared region included the SNL29A4, CDH23, PSAP, CHST3, DDIT4, CBARA1,
AP3M1, MYOZ1, PLAU, and ADK genes.

There is a high possibility that the $C D H 23, P S A P$, and $M Y O Z 1$ genes are related to hearing loss in this study. It is well known that mutations of $\mathrm{CDH} 23$ (cadherin-like 23) cause progressive non-syndromic hearing loss (DFNB12) in an autosomal recessive manner or of Usher syndrome type 1D, characterized by congenital, bilateral, profound sensorineural hearing loss, vestibular areflexia, and adolescent-onset retinitis pigmentosa (Becirovic et al., 2008). PSAP (prosaposin) is reported to help maintain normal innervation of the organ of corti in mice (Akil et al., 2006). In a study of the expression levels of candidate genes in the various mouse tissues, MYOZ1 showed higher expression in the inner ear than in other tissues of the mouse (data not shown). From the direct sequencing of these genes, we were unable to identify any disease-causing variants in the exons and exon-intron boundaries. The causative mutation may be located in the inter-exon areas of these three genes or in other candidate genes that map to this region. Further examination of this area may identify the gene responsible for ADNSHL.

The present study suggests a new possible causative region for hearing loss, with significant evidence for ADNSHL in the SNP-based linkage analysis. A few causative genes have been isolated in East Asian populations, and these will be valuable for understanding the genetic diagnosis of ADNSHL in this geographic area. Only five mutated genes for hearing loss have been identified in the Korean population, despite the significant genetic heterogeneity. Eleven deafness genes have been reported as being responsible for non-syndromic hereditary hearing loss in Japan (Usami et al., 2008). In China, the molecular etiology of six prominent deafnessrelated genes has been investigated, to provide genetic counseling for hearing-loss patients, although the molecular defects in about $66 \%$ of patients with non-syndromic hearing impairment in China remain to be identified (Yuan et al., 2009). Therefore, our findings provide valuable information for the genetic diagnosis and counseling of patients with hearing impairment in Korean and East Asian populations. We show that non-parametric linkage analysis with high-density SNPs is a useful technique. In the present study, SR-167 had very low power in the traditional analysis to detect LOD because all the available family members are affected. However, we managed to identify significant genetic loci using nonparametric linkage analysis with SNPs. Combining this method with whole-genome or exon sequencing may allow elucidation of the causal gene and mutations.

This work was supported by Korea National Institute of Health intramural research grant 4800-4845-300-210 (no. 2009N63001-00). 


\section{REFERENCES}

Abecasis, G. R., and Wigginton, J. E. (2005) Handling markermarker linkage disequilibrium: pedigree analysis with clustered markers. Am. J. Hum. Genet. 77, 754-767.

Abecasis, G. R., Cherny, S. S., Cookson, W. O., and Cardon, L. R. (2002) Merlin--rapid analysis of dense genetic maps using sparse gene flow trees. Nat. Genet. 30, 97-101.

Akil, O., Chang, J., Hiel, H., Kong, J. H., Yi, E., Glowatzki, E., and Lustig, L. R. (2006) Progressive deafness and altered cochlear innervation in knock-out mice lacking prosaposin. J. Neurosci. 26, 13076-13088.

Becirovic, E., Ebermann, I., Nagy, D., Zrenner, E., Seeliger, M. W., and Bolz, H. J. (2008) Usher syndrome type 1 due to missense mutations on both $\mathrm{CDH} 23$ alleles: investigation of mRNA splicing. Hum. Mutat. 29, 452.

Bitner-Glindzicz, M. (2002) Hereditary deafness and phenotyping in humans. Br. Med. Bull. 63, 73-94.

Cryns, K., and Van Camp, G. (2004) Deafness genes and their diagnostic applications. Audiol. Neurootol. 9, 2-22.

Evans, D. M., and Cardon, L. R. (2004) Guidelines for genotyping in genomewide linkage studies: single-nucleotidepolymorphism maps versus microsatellite maps. Am. J. Hum. Genet. 75, 687-692.

Gonzalez-Neira, A., Rosa-Rosa, J. M., Osorio, A., Gonzalez, E., Southey, M., Sinilnikova, O., Lynch, H., Oldenburg, R. A., van Asperen, C. J., Hoogerbrugge, N., et al. (2007) Genomewide high-density SNP linkage analysis of non-BRCA1/2 breast cancer families identifies various candidate regions and has greater power than microsatellite studies. BMC Genomics 8, 299.

John, S., Shephard, N., Liu, G., Zeggini, E., Cao, M., Chen, W.,
Vasavda, N., Mills, T., Barton, A., Hinks, A., et al. (2004) Whole-genome scan in a complex disease using 11,245 single-nucleotide polymorphisms: comparison with microsatellites. Am. J. Hum. Genet. 75, 54-64.

Kong, A., Gudbjartsson, D. F., Sainz, J., Jonsdottir, G. M., Gudjonsson, S. A., Richardsson, B., Sigurdardottir, S., Barnard, J., Hallbeck, B., Masson, G., et al. (2002) A highresolution recombination map of the human genome. Nat. Genet. 31, 241-247.

North, B. V., Curitis, D., and Sham, P. C. (2003) A note on calculation of empirical $\mathrm{P}$ values from Monte Carlo procedure. Am. J. Hum. Genet. 72, 498-499.

Schaid, D. J., Guenther, J. C., Christensen, G. B., Hebbring, S., Rosenow, C., Hilker, C. A., McDonnell, S. K., Cunningham, J. M., Slager, S. L., Blute, M. L., and Thibodeau, S. N. (2004) Comparison of microsatellites versus singlenucleotide polymorphisms in a genome linkage screen for prostate cancer-susceptibility loci. Am. J. Hum. Genet. 75, 948-965.

Usami, S., Wagatsuma, M., Fukuoka, H., Suzuki, H., Tsukada, K., Nishio, S., Takumi, Y., and Abe, S. (2008) The responsible genes in Japanese deafness patients and clinical application using Invader assay. Acta Otolaryngol. 128, 446-454.

Wigginton, J. E., and Abecasis, G. R. (2005) PEDSTATS: descriptive statistics, graphics and quality assessment for gene mapping data. Bioinformatics 21, 3445-3447.

Yuan, Y., You, Y., Huang, D., Cui, J., Wang, Y., Wang, Q., Yu, F., Kang, D., Yuan, H., Han, D., and Dai, P. (2009) Comprehensive molecular etiology analysis of nonsyndromic hearing impairment from typical areas in China. J. Transl. Med. 7, 79. 